\title{
Dispositivos móviles y realidad aumentada en el aprendizaje del alumnado universitario
}

\section{Mobile devices and augmented reality in the learning process of university students}

\author{
Julio Cabero Almenara \\ Bárbara Fernández Robles \\ Universidad de Sevilla (España) \\ Verónica Marín Díaz \\ Universidad de Córdoba, UCO (España)
}

\section{Resumen}

La Realidad Aumentada es una tecnología emergente que, cada día, tiene una mayor incidencia en la docencia. Tanto la Realidad Aumentada como la tecnología móvil se dibujan junto a ella; la tecnología móvil se dibuja como uno de los binomios más eficaces para apoyar un aprendizaje significativo y ubicuo. No obstante, para que este pueda funcionar con validez, el estudiante debe encontrarse motivado para utilizarla durante el proceso formativo. A través de la aplicación del modelo Instructional Material Motivational Survey de Keller (1983), se ha tratado de determinar el grado de motivación que el alumnado de Grado de Pedagogía de la Universidad de Sevilla ( $\mathrm{N}=148)$ tiene sobre la utilización en el aula de apuntes enriquecidos a través de la Realidad Aumentada y disponibles mediante dispositivos móviles. Mediante una aplicación diseñada para la materia, ha sido valorado de forma positiva por los alumnos participantes en este estudio. Además, podemos señalar que el principal hallazgo logrado ha sido la alta relación entre la motivación de los alumnos para emplear los apuntes enriquecidos y el rendimiento en la materia que los emplea. Igualmente, se ha constatado que la utilización de la Realidad Aumentada beneficia el proceso de aprendizaje. Por tanto, podemos concluir la validez y viabilidad del binomio con respecto a la obtención de una mejora de los resultados de aprendizaje del alumnado.

Palabras clave: tecnologías de la información y la comunicación; método de enseñanza; educación a distancia; telecomunicación.

\begin{abstract}
Augmented Reality is an emerging technology having more and more influence on teaching. Together with it, mobile technology is drawn as one of the most effective pairing to support a meaningful and omnipresent learning. However, for it to be able to work with validity, the student must be motivated to use them during the training process, so that the learning process can work with validity. By means of the application of the Instructional
\end{abstract}


Material Motivational Survey by Keller (1983), we have tried to determine the degree of motivation that students, from the Degree of Pedagogy at Seville University $(\mathrm{N}=148)$, have about the usage in the classroom of notes enriched through Augmented Reality and available in mobile devices. Through an application designed exclusively for the subject, it has been valued in a positive way by the students who participate in this research. Moreover, we can highlight that the main finding achieved has been the narrow relationship between the students' motivation to use the enriched notes and the degree of success in the subject when using them. Likewise, we can confirm that Augmented Reality benefits the learning process. Therefore, we conclude the validity and viability of such a paring related to the achievement of an improvement in the students' learning results.

Keywords: information and communication technologies; teaching method; distance telecommunication.

La Realidad Aumentada (Augmented Reality, RA) se está convirtiendo en una tecnología emergente con grandes posibilidades para su uso educativo (Bacca, Baldiris, Fabregat, Graf y Kinshuk, 2014; Tecnológico de Monterrey, 2015; Johnson, Adams, Cummins, Estrada, Freeman y Hall, 2016; Cabero y Barroso, 2016; Cabero y García, 2016), y ello se debe, entre otros motivos, a la facilidad con la que esta permite acceder a la información, ya que normalmente se accede gracias a los dispositivos móviles; recursos, que no lo olvidemos, poseen una alta presencia en los estudiantes universitarios en el contexto iberoamericano (Sevilla y Vázquez, 2015).

Esta evolución, tanto en la RA como en los dispositivos móviles, podemos observarla también cuando utilizamos la herramienta Ngram Viewer creada para Google, que facilita la evolución de determinados términos en los documentos digitalizados en Google. Esta señala que el avance es casi similar de los términos: realidad aumentada/augmented reality y servicios móviles/mobile devices.

Ahora bien, ¿qué es la realidad aumentada? Podemos decir que es una tecnología que permite la combinación de información digital e información física en tiempo real, por medio de distintos soportes tecnológicos, como, por ejemplo las tablets o los smartphones, y que permite crear, con ello, una nueva realidad (Morales, Benítez, Silva, Altamira y Mendoza, 2015).

Esta integración mixta de la realidad física y la digital se puede llevar a cabo en diferentes niveles, que van desde la utilización de los códigos QR, el uso de imágenes, la utilización de objetos en 3D, la movilización de coordenadas mediante GPS, hasta las huellas termales (Redondo, Sánchez, Narcis y Regot, 2012; Cabero y García, 2016). Por otra parte, los sistemas de RA pueden clasificarse según su ubicación o según las imágenes.

Los sistemas de RA basados en la localización utilizan los datos sobre la posición de los dispositivos móviles, determinado por el Sistema de Posicionamiento Global (GPS) o Sistemas de posicionamiento basados en Wi-Fi, etc. En contraste, la RA basada en imágenes se centra en técnicas de reconocimiento de imagen utilizadas para determinar la posición de objetos físicos en el entorno real para la ubicación 
apropiada de los contenidos virtuales relacionados con estos objetos (Wojciechowski y Cellary, 2013, p. 572).

Su significación para la formación viene determinada, por una parte, por sus características específicas, como son: ser una realidad mixta, la posibilidad que ofrece para integrar en tiempo real tanto diferentes capas de información como distintos tipos de formatos (textos, url, vídeos, etc.), es una tecnología interactiva, su facilidad de manejo, y que, mediante su utilización, enriquecemos o alteramos la información de la realidad añadiéndole información adicional (Cabero y García, 2016). Y por otra, porque los dispositivos que se suelen utilizar para su observación, como son los Smartphones, son tecnologías de las que disponen con facilidad los alumnos universitarios.

Su utilización en la formación, como señalan diferentes autores (Wu, Lee, Chang y Liang, 2013; Carozza, Tingdahl y Gool, 2014; Cubillo, Martín, Castro y Colmenar, 2014; Jeřábek, Rambousek y Wildová; 2014; Barba, Yasaca y Manosalvas, 2015; Jamali, Fairuz, Wai y Oskam, 2015; Fonseca, Redondo y Valls, 2015; Han, Jo, Hyun y So, 2015; Santos, Wolde, Taketomi, Yamamoto, Rodrigo, Sandor y Kato, 2016), permite diferentes posibilidades, por ejemplo: a) eliminar información que pueda entorpecer la captación de la información significativa por el estudiante; b) aumentar o enriquecer la información de la realidad para hacerla más comprensible al estudiante; c) poder observar un objeto desde diferentes puntos de vista, seleccionando, el estudiante, el momento y posición de observación; d) potenciar el aprendizaje ubicuo; e) crear escenarios "artificiales" seguros para los estudiantes como pueden ser laboratorios o simuladores; f) enriquecer los materiales impresos para los estudiantes con información adicional en diferentes soportes; g) y convertir a los alumnos en "proconsumidores" de objetos de aprendizaje en formato RA. A estas características debemos incorporarles que puede ser utilizada en diferentes disciplinas y en distintos niveles educativos (De Pedro Carracedo y Méndez, 2012; Bressler y Bodzin, 2013; Prendes, 2015; Cabero y García, 2016), aunque se debe reconocer que es en la formación universitaria donde se está utilizando con mayor frecuencia (Lin, Been-Lirn, Li, Wang y Tsai, 2013).

Sobre su impacto en la educación, y aunque todavía las investigaciones realizadas son limitadas, y ello posiblemente sea uno de los grandes problemas que tenga para su incorporación a la enseñanza, sí han puesto de manifiesto algunos aspectos como son que los alumnos muestran actitudes favorables hacia ella y que su utilización aumenta la motivación hacia el aprendizaje (Bressler y Bodzin, 2013; Kamarainen, Metcalf, Grotzer, Browne, Mazzuca, Tutwiler y Dede, 2013; Di Serio, Ibáñezy Delgado, 2013; Cózar, De Moya, Hernández y Hernández, 2015), que favorecen la creación de un contexto constructivista de formación (Chen y Tsai, 2012; Wojciechowski y Cellary, 2013), que propicia un entorno activo de enseñanza (Fombona, Pascual y Madeira, 2012), que despierta un elevado grado de satisfacción en los alumnos (Han et al., 2015; Kim, Hwang y Zo, 2016), y que su utilización mejora los resultados de aprendizajes (Bongiovani, 2013; Chang, Wu y Hsu, 2013; Kamarainen et al., 2013). 


\title{
LA MOTIVACIÓN HACIA EL USO DE LAS TECNOLOGÍAS EMERGENTES
}

La motivación ha sido siempre considerada como una de las variables que se ha percibido como determinante para que la persona realice una acción o conducta. Keller (1983, p. 389) se refiere a ella como

\begin{abstract}
la magnitud y dirección de la conducta. En otras palabras, se refiere a la elección que la persona hace en cuanto a lo que experimenta o las metas a las que se acercará o evitará, y el grado del esfuerzo que va a ejercer en ese aspecto. La motivación está influenciada por miradas internas y características externas. Las personas responden a su contexto sobre las bases de reflexiones internas, impulsos, percepciones y metas, y sobre la base percibida y oportunidades reales, y el refuerzo del contexto externo.
\end{abstract}

En esta línea, Cheng y Yeh (2009) llaman la atención respecto a que es un estado interno o condición que despierta a la acción, dirige y persiste en nuestro comportamiento, e involucra en ciertas actividades, y que, en el contexto de aula, se refiere a "experiencias subjetivas. En particular, la buena disposición de los estudiantes a participar en actividades de clase y sus razones para hacerlo" (Cheng y Yeh, 2009, p. 597).

Específicamente situado en el terreno del diseño instruccional, Keller, a lo largo de diferentes trabajos $(1983,1987,2010)$, ha formulado su modelo ARCS, a través del cual señala que la motivación, en contextos instruccionales, viene determinada por la interacción de cuatro dimensiones: la atención (A), la relevancia (R), la confianza (C) y la satisfacción (S) (http://www.arcsmodel.com/). La atención conduce a la relevancia y esta a la confianza y todas juntas a la satisfacción, todo ello dentro del ámbito de la motivación continuada para aprender.

Para Keller (2010), la categoría de atención incluye características humanas, tales como el reflejo orientación, la curiosidad y la búsqueda de sensaciones; la relevancia, se refiere a aquellas cosas que la persona percibe como un instrumento para satisfacer las necesidades y la satisfacción personal, incluyendo el cumplimiento de los objetivos personales; la confianza, que, como señala el propio autor, es un concepto complejo que abarca varios constructos motivacionales, que van desde aquellos que explican las percepciones de control personal y la esperanza para el éxito en el extremo opuesto a la impotencia; siendo el paso final, en el proceso motivacional, crear satisfacción por lo que se continuará la motivación para aprender.

El modelo ilustra como la curiosidad de una persona ("atención") y los motivos o valores ("relevancia"), combinados con su esperanza de éxito ("confianza") determinarán qué objetivos tiene la más alta prominencia y, por tanto, dará lugar a esfuerzo intencional para lograr la meta. Las influencias ambientales tales como el entusiasmo del maestro y los valores sociales, la calidad de la instrucción, la claridad de expectativas y la disponibilidad de recursos también influirán dirigido a 
un objetivo esfuerzo y plomo hacia algún grado de cumplimiento, o el rendimiento, lo que lo hará también ser influenciados por los propios conocimientos, capacidad y habilidades (Keller, 2008).

Por tanto, la base en la que se apoya es, que si la estrategia de la instrucción se percibe como útil para la realización de las metas, es importante para el estudiante; entonces es probable que este aprenda los contenidos y adquiera las competencias.

El modelo de Keller que, como señalan Loorbach, Peters, Karreman y Steehouder (2015, p. 2004),

se ha utilizado innumerables veces para aplicar estrategias de motivación para materiales de instrucción, y para poner a prueba sus efectos. Aunque el modelo fue originalmente diseñado para influir en la motivación del estudiante en un entorno de aprendizaje clásico, con la interacción cara a cara entre el profesor y los estudiantes, también ha sido aplicado y probado a fondo para en otros ajustes, como la enseñanza asistida por ordenador, y la educación virtual y a distancia.

Para su diagnóstico, Keller (2010) elaboró un instrumento denominado Instructional Material Motivational Survey (IMMS), el cual está compuesto por 35 ítems, que recogían información de las 4 dimensiones que lo conforman: atención (12 ítems), confianza (9 ítems), relevancia (9 ítems) y satisfacción (5 ítems). El instrumento está elaborado con construcción tipo Likert, y con siete opciones de respuesta, desde 1=Extremadamente improbable/en desacuerdo a $7=$ Extremadamente probable/de acuerdo.

El modelo ha sido aplicado para indagar sobre el grado de motivación que despiertan diferentes medios: videojuegos (Proske, Roscoe y McNamara, 2014), la incorporación de vídeo en sesiones de formación en e-learning (Che, 2012), podcast (Bolliger, Supanakorn y Boggs, 2010), o la utilización del MOOC (Castaño, Maiz y Garay, 2015). Señalar que se ha utilizado también para conocer el grado de motivación que los objetos de RA despiertan en los alumnos (Di Serio, Ibáñez y Delgado, 2013; Lu y Ying-Chieh, 2014; Wei, Weng, Liu y Wang, 2015; Lu y Liu, 2015).

\section{LA INVESTIGACIÓN REALIZADA: OBJETIVOS, DISEÑO, MUESTRA E INSTRUMENTOS DE RECOGIDA DE INFORMACIÓN}

\section{Objetivos de la investigación}

La investigación se desarrolló en el curso académico 2015-16, y perseguía los siguientes objetivos generales:

a. Conocer el grado de motivación medido mediante el IMMS de Keller y de las dimensiones que lo conforman (atención, renovación, relevancia y satisfacción), que la utilización de apuntes enriquecidos con objetos de RA despertaban en 
los estudiantes, y si tal grado de motivación repercutía en la adquisición de conocimientos.

b. Analizar si existían diferencias significativas en el rendimiento alcanzado en los estudiantes tras la interacción con apuntes enriquecidos mediante RA.

c. Conocer las valoraciones que los alumnos universitarios hacían de los objetos producidos en RA, y si estas repercutían en el rendimiento alcanzado.

d. Analizar si la evaluación realizada por parte del estudiante universitario del objeto producido en RA repercutía en el grado de motivación (atención/renovación/ relevancia/satisfacción) medidos mediante el IMMS de Keller.

Para el alcance de estos objetivos formulamos diferentes hipótesis, que seguían la siguiente estructura:

- Ho (hipótesis nula): No hay diferencias significativas con un riesgo alto de equivocarnos del 0.05 respecto a las puntuaciones alcanzadas por los alumnos en el pretest y el postest; entre la motivación alcanzada en el instrumento del IMMS y el rendimiento; $y$ entre el rendimiento y las puntuaciones alcanzadas en la evaluación que los alumnos realizaron del objeto producido en RA.

- H1 (hipótesis alternativa): Hay diferencias significativas con un riesgo alto de equivocarnos del 0.05 respecto a las puntuaciones alcanzadas por los alumnos en el pretest y el postest; entre la motivación alcanzada en el instrumento del IMMS y el rendimiento; y entre el rendimiento y las puntuaciones alcanzadas en la evaluación que los alumnos realizaron del objeto producido en RA.

\section{Diseño de la investigación}

En función de los objetivos planteados, se adoptó la decisión de utilizar un diseño pretest-postest con un grupo experimental conformado por dos grupos de clases naturales que recibieron el mismo tratamiento, es decir, la interacción con el objeto de RA producido. En la tabla 1 se presenta el proceso seguido.

Tabla 1. Estructura del diseño utilizado

\begin{tabular}{|c|c|c|c|}
\hline Grupos & Pretest & Tratamiento & Postest \\
\hline $\begin{array}{l}\text { A (formado } \\
\text { por dos grupos } \\
\text { naturales de } \\
\text { clase) }\end{array}$ & $\begin{array}{l}\text { Prueba de } \\
\text { rendimiento }\end{array}$ & $\begin{array}{l}\text { Interacción con el objeto } \\
\text { de RA (explicación del } \\
\text { funcionamiento del } \\
\text { objeto, toma de contacto } \\
\text { del alumno con la guía, } \\
\text { e interacción individual } \\
\text { del alumno con el objeto } \\
\text { durante dos semanas }\end{array}$ & $\begin{array}{l}\text { Prueba de rendimiento. } \\
\text { IMMS de Keller } \\
\text { Instrumento de } \\
\text { evaluación de la calidad } \\
\text { del objeto en RA }\end{array}$ \\
\hline
\end{tabular}


Aunque es un diseño considerado del tipo pre-experimental (Sans, 2012), presenta la ventaja de la facilidad de su control por parte del investigador, y además, al llevarse a cabo en dos grupos naturales el control de determinadas "variables extrañas", estaba repartido por el azar de asignación al grupo por la Administración del Centro. Por otra parte, en el terreno de la investigación educativa, los estudios comparativos del control no han demostrado su significación para la evaluación de las tecnologías (Barroso y Cabero, 2010); sin olvidarnos que no hubiera sido posible llevar a cabo la evaluación del objeto en RA.

\section{La muestra}

La muestra utilizada en la investigación es no probabilística, de conveniencia o causal (Alamino, 2006; Sabariego, 2012), que es aquella que se basa en la facilidad de acceso por parte del investigador a los sujetos que participan en la misma. En nuestro caso, eran estudiantes que estaban estudiando la asignatura de "Tecnología Educativa" de segundo curso del Grado de Pedagogía, impartido en la Facultad de Ciencias de la Educación de la Universidad de Sevilla, que era impartida por profesores asociados al proyecto de investigación donde se inserta el estudio que se presenta. El número total de participantes fue de 148, de los cuales el $24.34 \%$ ( $\mathrm{f}=36$ ) eran hombres y el $75.68 \%$ ( $\mathrm{f}=112)$ mujeres.

\section{El material producido}

La experiencia versó sobre los contenidos de las "formas de utilizar el vídeo en los procesos de enseñanza-aprendizaje". Y para ello, se realizó una versión impresa que fue enriquecida con clip de vídeos (figura 1), para que fueran observados por los estudiantes a través de diferentes dispositivos móviles (Smartphone o Tablet), donde se trataban distintos aspectos relacionados con la temática expuesta, que iban desde las diversas formas de utilizarlos hasta la significación de las guías didácticas para la contextualización de los video-mensajes. Además, en los apuntes facilitados a los estudiantes, se incluía una bibliografía temática, referencias para saber más y una parte, a manera de "tutorial", en la cual se les explicaba a los alumnos el procedimiento que debían seguir para descargarse la APP correspondiente e instalarla en su dispositivo móvil, y la forma en la cual deberían utilizar estos para acceder a la información (figura 4). Señalar que, en la primera sesión, se les ofreció la dirección Web (http://intra.sav.us.es/proyectorafodiun/images/pdf/objetos-ra/ Roles video-lanzador.pdf) en la cual podían obtener los apuntes para que fueran impresos. 
Figura 1. Imagen de uno de los clips de vídeos

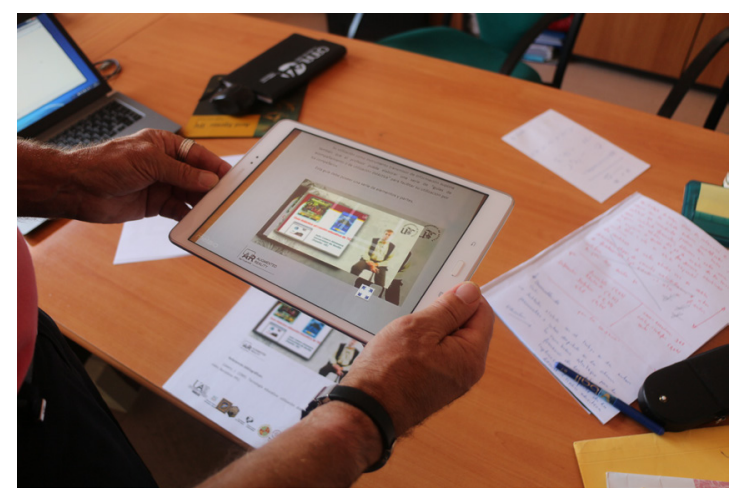

Figura 2. Presentación de los apuntes

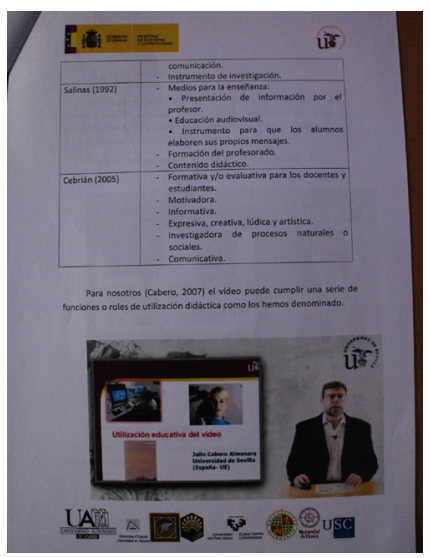

En su incorporación a la enseñanza se siguieron diferentes fases:

1. Cumplimentación de la prueba de pretest.

2. Explicación por los profesores del funcionamiento del objeto, el lugar desde el cuál deberían descargarse la APP para instalarla en sus dispositivos móviles y los apuntes.

3. Período de dos semanas de trabajo de los estudiantes con el objeto producido con sus dispositivos móviles. Aclaración en las clases de prácticas de la asignatura de las dudas que se les presentaban a los estudiantes.

4. Cumplimentación de los instrumentos de diagnóstico de la motivación, el rendimiento y la calidad del objeto producido. 
Desde el comienzo de la experiencia, los estudiantes fueron informados de que las calificaciones obtenidas se tendrían en cuenta para las calificaciones de la asignatura.

Indicar que, para superar el efecto de novedad de la tecnología, que puede repercutir en los resultados alcanzados (Barroso y Cabero, 2010), los alumnos trabajaron en sesiones previas con objetos producidos en RA.

\section{Instrumentos de recogida de información}

Los instrumentos de recogida de información fueron tres: prueba de elección múltiple para el análisis de rendimiento obtenido por los estudiantes tras la interacción, el Instructional Material Motivational Survey (IMMS) elaborado por Keller (2010) para el análisis de la motivación de los alumnos hacia la participación en la experiencia, y un instrumento elaborado "ad hoc" para que el alumnado evaluara los apuntes enriquecidos elaborados.

Para la evaluación de la calidad de los apuntes enriquecidos en RA se elaboró un instrumento "ad hoc", como ya se ha indicado, para esta investigación a partir de los elaborados por diferentes autores (Cabero y Llorente, 2009; Cabero y Marín, 2013; Marín, Cabero y Barroso, 2014), en otras investigaciones para la evaluación de objetos de aprendizaje de materiales multimedia, de materiales para la formación bajo la modalidad en e-learning, y de objetos producidos para un entorno virtual de formación bajo la estructura de "Entorno Personal de Aprendizaje". El instrumento estaba constituido por 12 ítems, que perseguían recoger información de diferentes dimensiones: aspectos técnicos y estéticos (4 ítems), facilidad de navegación y desplazamiento por el entorno (6 ítems) y dos ítems destinados a la evaluación de la guía/tutorial del programa. El instrumento se construyó con formato tipo Likert con 6 opciones de respuesta (1=muy negativo/muy en desacuerdo y $6=$ muy positivo/ muy de acuerdo).

Para la obtención del índice de fiabilidad de ambos instrumentos, aplicamos la prueba alfa de Cronbach, por ser uno de los estadísticos más apropiados para obtenerla con este tipo de instrumentos ( $\mathrm{O}^{\prime}$ Dwyer y Bernauer, 2014). Tras su aplicación, se obtuvieron los valores para ambos instrumentos y las dimensiones que lo conforman que presentamos en la tabla 2.

Tabla 2. Fiabilidad de los instrumentos y de las dimensiones que lo conforman

\begin{tabular}{|l|l|l|l|}
\hline \multicolumn{2}{|c|}{ IMMS } & \multicolumn{1}{c|}{ Evaluación de la calidad del objeto } \\
\hline Dimensión & Valor alfa & Dimensión & Valor alfa \\
\hline Total instrumento & 0.928 & Total instrument & 0.930 \\
\hline Atención & 0.849 & Aspectos técnicos & 0.888 \\
\hline Confianza & 0.751 & Facilidad de navegación & 0.860 \\
\hline
\end{tabular}


Los valores obtenidos, de acuerdo con diferentes autores (Mateo, 2004; O' Dwyer y Bernauer, 2014), denotarían un elevado nivel de fiabilidad de la globalidad del instrumento y de las diferentes dimensiones que lo conforman.

El instrumento creado para el rendimiento académico se elaboró bajo la modalidad de dos pruebas de elección múltiple ("pretest" y "postest"), y estaban constituidas por 15 ítems, que recogían información de las siguientes categorías de la taxonomía de Bloom: aplicar ( 4 ítems), recordar (6 ítems) y comprender (5 ítems); siendo los ítems similares para las dos modalidades, aunque alterando su orden de presentación.

Los instrumentos se administraron vía Internet y se encuentran en la siguiente dirección web: http://www.sav.us.es/encuestas/rafodiun/video/postest IMMS. $\underline{\mathrm{html}}$

\section{ANÁLISIS DE LOS RESULTADOS}

Para facilitar la comprensión de los resultados alcanzados, en la tabla 2 presentamos los valores medios obtenidos en el instrumento IMMS, en el elaborado para el rendimiento y el destinado a la evaluación del material por parte de los estudiantes.

Tabla 3. Valores medios y desviaciones típicas alcanzadas con los instrumentos del IMMS y de valoración de la calidad técnica, y las pruebas de rendimiento

\begin{tabular}{|l|l|l|l|l|l|l|l|l|}
\hline \multicolumn{3}{|c|}{ RENDIMIENTO } & \multicolumn{3}{c|}{ Calidad técnica } \\
\hline Dimensión & M & D.tp. & Dimensión & M & D.tp. & Dimensión & M & D.tp. \\
\hline Pretest & 4.37 & 3.39 & $\begin{array}{l}\text { Global } \\
\text { IMMS }\end{array}$ & 4.08 & 0.58 & $\begin{array}{l}\text { Global } \\
\text { instrument }\end{array}$ & 3.60 & 0.82 \\
\hline Postest & 10.43 & 2.91 & Confianza & 3.91 & 0.62 & $\begin{array}{l}\text { Aspectos } \\
\text { técnicos }\end{array}$ & 3.66 & 0.86 \\
\hline & & Atención & 4.00 & 0.62 & $\begin{array}{l}\text { Facilidad de } \\
\text { navegación }\end{array}$ & 3.46 & 0.87 \\
\hline & & & Satisfacción & 4.39 & 1.01 & $\begin{array}{l}\text { Tutorial del } \\
\text { programa }\end{array}$ & 3.66 & 0.95 \\
\hline
\end{tabular}

Un breve análisis de la tabla anterior permite señalar diferentes aspectos: por una parte, y con respecto al rendimiento, las puntuaciones del postest son superiores a las 
obtenidas inicialmente, lo que denotaría cierto grado de adquisición de información, y por otra parte, que las puntuaciones medias en los otros dos instrumentos superan los valores medios de las escalas ofrecidas, 3.5 para el instrumento del IMMS y de 3 para el de la calidad del material producido.

Mostrados los valores medios, pasaremos a analizar las diferentes hipótesis que formulamos anteriormente, y para ello, comenzaremos analizando si las diferencias entre las puntuaciones alcanzadas entre el pretest y postest eran significativas desde un punto de vista estadístico, aplicando para el contraste de las Ho y $\mathrm{H} 1$ la prueba T de Student obteniendo un valor de 16.107 con un nivel de significación del 0.000 para 147 grados de libertad, lo que permite rechazar las HO formuladas con un riesgo inferior al 0.01; y en consecuencia, podemos señalar que la participación de los alumnos en la experiencia de enriquecimiento de apuntes mediante la RA, sirvió para que adquirieran los contenidos presentados y, por tanto, para que aumentaran su aprendizaje.

Para analizar las hipótesis formuladas referidas a si la motivación mostrada por el estudiante, y el nivel expuesto en la confianza, atención, satisfacción y relevancia analizadas mediante el IMMS, repercutía en el rendimiento alcanzado tras la interacción con el objeto de RA, aplicamos el coeficiente de correlación de Pearson, obteniendo los valores que presentamos en la tabla 4.

Tabla 4. Correlaciones entre el rendimiento y la motivación mostrada por el estudiante, y el nivel mostrado en la confianza (C), atención (A), satisfacción (S) y relevancia (R)

\begin{tabular}{|l|l|c|c|c|c|c|}
\hline \multirow{3}{*}{ Rendimiento } & \multicolumn{1}{c}{ Global } & \multicolumn{1}{c|}{ C } & \multicolumn{1}{c|}{ A } & \multicolumn{1}{c|}{ S } & \multicolumn{1}{c|}{ R } \\
\cline { 2 - 7 } & C.C. Pearson & $\left.0.243^{(* *}\right)$ & $0.328\left(^{* *}\right)$ & $0.166\left(^{*}\right)$ & $0.217\left(^{* *}\right)$ & $0.164\left(^{*}\right)$ \\
\cline { 2 - 7 } & Sig. bilateral & 0.003 & 0.000 & 0.046 & 0.008 & 0.048 \\
\hline
\end{tabular}

Nota. ${ }^{*}=$ significativa al $0.05 \mathrm{y}^{* *}=$ significativa a 0.01 .

Como indican Etxeberria y Tejedor (2005) a la hora de analizar las puntuaciones alcanzadas mediante el coeficiente de correlación de Pearson, debemos fijarnos en diferentes aspectos, como son: si la relación es directa o inversa, que se refleja en el signo y su grado de relación, que aumentará conforme aumente el valor obtenido.

Observando los valores presentados en la tabla 4, en todos los casos permiten señalar: que las relaciones entre las diferentes variables contrastadas, rendimiento y motivación (confianza-atención-satisfacción-relevancia), son directas, es decir, cuando una aumenta, la otra también lo hace; que las relaciones son moderadas, destacando la encontrada entre el rendimiento y dimensión confianza mediada por el IMMS de Keller; y que todas son significativas al nivel del 0.05 o inferior, por tanto, podríamos rechazar la Ho referida a la no existencia de relaciones y aceptar la H1.

Continuando con los resultados alcanzados con el rendimiento, nuestro siguiente bloque de hipótesis iba destinado a analizar si existían relaciones significativas entre 
el rendimiento académico y las puntuaciones alcanzadas en la evaluación que los alumnos realizaron del objeto producido. De nuevo, el estadístico utilizado para ello fue el coeficiente de correlación de Pearson. En la tabla 5 presentamos los resultados alcanzados.

Tabla 5. Correlaciones entre el rendimiento y la motivación mostrada por el estudiante, y el nivel mostrado en la confianza (C), atención (A), satisfacción (S) y relevancia (R)

\begin{tabular}{|l|l|l|l|l|l|}
\hline \multicolumn{2}{|c|}{} & $\begin{array}{c}\text { Calidad } \\
\text { técnica }\end{array}$ & $\begin{array}{c}\text { Aspectos } \\
\text { técnicos }\end{array}$ & $\begin{array}{c}\text { Facilidad de } \\
\text { navegación }\end{array}$ & Tutorial \\
\hline \multirow{2}{*}{ Rendimiento } & C.C. Pearson & 0.142 & $0.173\left({ }^{*}\right)$ & 0.070 & $0.216\left(^{* *}\right)$ \\
\cline { 2 - 7 } & Sig. bilateral & 0.088 & 0.038 & 0.403 & 0.009 \\
\hline
\end{tabular}

Nota. ${ }^{*}=$ significativa al $0.05 \mathrm{y}^{* *}=$ significativa a 0.01 .

Como podemos observar en la tabla 5 , los valores obtenidos señalan que las relaciones entre el rendimiento y la evaluación realizada por el sujeto del objeto en RA, con el que interaccionaron los estudiantes, son de carácter positivo, por tanto, podemos decir que cuando una aumenta, la otra también lo hace; las relaciones son muy débiles en la mayoría de los casos; y no en todos los casos se han mostrado significativas.

Para finalizar, vamos a presentar los resultados obtenidos con el coeficiente de correlación de Pearson, cuando se analizaron las relaciones que se podían establecer entre la evaluación que los alumnos realizaron del objeto en RA, con el cual interaccionaron y las puntuaciones mostradas en el IMMS y en las diferentes dimensiones que lo conformaban. En la tabla 6, presentamos los resultados alcanzados.

Tabla 6. Coeficiente correlación de Pearson entre la calidad técnica y el IMMS

\begin{tabular}{|l|l|l|l|l|l|}
\hline \multicolumn{2}{|c|}{} & \multicolumn{1}{|c|}{$\begin{array}{c}\text { Calidad } \\
\text { técnica }\end{array}$} & $\begin{array}{c}\text { Aspectos } \\
\text { técnicos }\end{array}$ & $\begin{array}{c}\text { Facilidad } \\
\text { de } \\
\text { navegación }\end{array}$ & $\begin{array}{c}\text { Tutorial del } \\
\text { programa }\end{array}$ \\
\hline \multirow{2}{*}{ IMMS } & C.C. Pearson & $\left.0.543^{(* *}\right)$ & $0.562\left(^{* *}\right)$ & $0.524\left(^{* *}\right)$ & $0.601\left({ }^{* *}\right)$ \\
\cline { 2 - 6 } & Sig. bilateral & 0.000 & 0.000 & 0.000 & 0.000 \\
\hline \multirow{2}{*}{ Confianza } & C.C. Pearson & $0.503\left(^{* *}\right)$ & $0.498\left(^{* *}\right)$ & $\left.0.477^{* *}\right)$ & $\left.0.652^{* *}\right)$ \\
\cline { 2 - 6 } & Sig. bilateral & 0.000 & 0.000 & 0.000 & 0.000 \\
\hline \multirow{2}{*}{ Atención } & C.C. Pearson & $\left.0.544^{* *}\right)$ & $0.548\left(^{* *}\right)$ & $\left.0.554^{* *}\right)$ & $\left.0.531^{* *}\right)$ \\
\cline { 2 - 6 } & Sig. bilateral & 0.000 & 0.000 & 0.000 & 0.000 \\
\hline
\end{tabular}




\begin{tabular}{|l|l|l|l|l|l|}
\hline \multicolumn{2}{|c|}{} & \multicolumn{1}{|c|}{$\begin{array}{c}\text { Calidad } \\
\text { técnica }\end{array}$} & $\begin{array}{c}\text { Aspectos } \\
\text { técnicos }\end{array}$ & $\begin{array}{c}\text { Facilidad } \\
\text { de } \\
\text { navegación }\end{array}$ & $\begin{array}{c}\text { Tutorial del } \\
\text { programa }\end{array}$ \\
\hline \multirow{2}{*}{ Satisfacción } & C.C. Pearson & $\left.0.513^{* *}\right)$ & $0.504\left(^{* *}\right)$ & $0.459\left(^{* *}\right)$ & $\left.0.527^{* *}\right)$ \\
\cline { 2 - 6 } & Sig. bilateral & 0.000 & 0.000 & 0.000 & 0.000 \\
\hline \multirow{2}{*}{ Relevancia } & C.C. Pearson & $0.375\left(^{* *}\right)$ & $0.404(* *)$ & $0.306\left(^{* *}\right)$ & $\left.0.395^{* *}\right)$ \\
\cline { 2 - 7 } & Sig. bilateral & 0.000 & 0.000 & 0.000 & 0.000 \\
\hline
\end{tabular}

Nota. ${ }^{*}=$ significativa al $0.05 ;{ }^{* *}=$ significativa al 0.01 .

Como podemos observar, los valores alcanzados indican diferentes aspectos: que las relaciones entre las diferentes variables contrastadas, valoración del objeto en RA y motivación (confianza-atención-satisfacción-relevancia), son directas y, por tanto, las dos aumentan progresivamente; que las relaciones entre ambas variables son aceptables y además de las más altas de cuantas se han realizado en el estudio; y que todas las correlaciones son significativas al nivel del $0.001 \mathrm{o}$ inferior.

\section{DISCUSIÓN Y CONCLUSIONES}

Para presentar las conclusiones del trabajo, se comenzará relacionándolas con los objetivos formulados. Y lo primero a señalar es que la motivación que despiertan los apuntes enriquecidos con objetos de RA, la podemos considerar significativa, tanto de forma general, como en la atención, la confianza, la relevancia y la satisfacción que despertó en los estudiantes. En líneas generales, los hallazgos encontrados coinciden con los obtenidos por otros investigadores, cuando los alumnos interaccionaron con diferentes objetos de aprendizaje en RA (Bressler y Bodzin, 2013; Kamarainen et al., 2013; Di Serio, Ibáñez y Delgado, 2013; Cubillo et al., 2014; Cózar et al., 2015); si bien aquí se han obtenidos en contextos de formación reglada y universitaria.

También indicar que el objeto producido en RA fue valorado positivamente por los estudiantes, tanto en lo que se refiere a su correcto funcionamiento técnico, como a la información presentada, y su facilidad de manejo y desplazamiento por el mismo. Destacar que lo que hemos denominado como "tutorial" del programa, es decir, el documento que pretendía explicar a los alumnos el procedimiento que debían seguir para descargar e instalar la APP, y la forma en la cual deberían utilizar los dispositivos móviles para acceder a la información, fue valorada de manera positiva por los estudiantes; documento que no se ha incorporado en otros estudios y que pensamos que es relevante para una correcta utilización de estos objetos de aprendizaje y para evitar la desorientación por los estudiantes en sus primeras utilizaciones. Nuestros resultados coinciden con los mostrados en otros estudios, respecto a que no son objetos difíciles de utilizar por los estudiantes; y por esta 
razón, es importante señalar la necesidad de elaborar tutoriales que faciliten la comprensión de cómo debe desenvolverse con estos objetos.

Respecto al rendimiento, tenemos que señalar que los alumnos mejoraron significativamente las puntuaciones en la prueba de conocimiento que, inicialmente, se les había administrado para conocer los conocimientos previos que tenían respecto a las formas de utilizar el vídeo en la enseñanza. Podemos decir que los resultados alcanzados indican que la utilización de apuntes enriquecidos con objetos de RA puede facilitar el aprendizaje y la adquisición de conocimiento por parte de los estudiantes. Ello va en la dirección apuntada por diferentes autores de que la construcción de libros en RA puede ser de gran utilidad para la formación (Dünser, Walker, Horner y Bentall, 2012; Lin, Been-Lirn, Li, Wang y Tsa, 2013; Gazcón, 2015; Mehmet, 2016), y abre fuertes posibilidades para la educación a distancia, al recibir los estudiantes información complementaria por esta tecnología que le facilitará la comprensión de los contenidos, y hará más fácil la adquisición del rendimiento.

El estudio ha puesto de manifiesto que hay una relación significativa entre la motivación que la utilización de los apuntes enriquecidos con objetos de RA ha despertado en los estudiantes, y el rendimiento que lograron. A mayor motivación (confianza, atención, satisfacción y relevancia) despertada por los estudiantes, mayor rendimiento alcanzaron. Idénticamente ocurrió con la relación entre la motivación y la evaluación que realizaron los estudiantes del objeto.

Ha resultado llamativo que no se encontraron relaciones significativas entre la evaluación que los alumnos hicieron del objeto y el rendimiento. La inexistencia de relaciones significativas podría explicarse por el hecho de que los alumnos, al saber que la puntuación obtenida en la prueba de rendimiento le servirían para su calificación final, se olvidaban cognitivamente de la percepción que tenían del medio e invertían esfuerzo para capturar la información y su significado. De todas formas, sería conveniente la profundización con estudios complementarios de esta variable.

Otra de las aportaciones del estudio se refiere a la fiabilidad de los instrumentos utilizados para el diagnóstico de las variables contempladas, mostrando índices de fiabilidad similares a los alcanzados en otras investigaciones (Huang, Huang y Tschopp, 2010; Keller, 2010; Che, 2012; Di Serio, Blanca y Delgado, 2013; Proske, Roscoe y McNamara, 2014).

Para finalizar, indicar que nuestro trabajo aporta referencias científicas para justificar la incorporación de apuntes enriquecidos con RA en la formación universitaria, donde además de despertar la motivación y la satisfacción por parte de los estudiantes, facilita que adquieran los contenidos presentados por ella.

Como desarrollos futuros de investigaciones, se proponen las siguientes actuaciones: replicar el estudio con apuntes enriquecidos que contengan objetos de diferente tipología y no solo clip de vídeos, trabajar con contenidos diferentes al utilizado en el presente estudio y trabajar con disciplinas científicas diferentes a Ciencias de la Educación. 


\section{Financiamiento}

El trabajo se enmarca dentro de un proyecto de investigación I+D financiado por el Ministerio de Economía y Competitividad del Gobierno de España denominado: "Realidad aumentada para aumentar la formación, diseño, producción y evaluación de programas de realidad aumentada para la formación universitaria" (EDU-5746-P - Proyecto Rafodiun).

\section{REFERENCIAS BIBLIOGRÁFICAS}

Alaminos, A. (2006). El muestreo en la investigación social. En A. Alaminos y J. L. Castejón, Elaboración, análisis e interpretación de encuestas, cuestionarios y escalas de opinión (4667). Alcoy: Marfil.

Bacca, J., Baldiris, S., Fabregat, R., Graf, S., y Kinshuk, G. (2014). Augmented Reality Trends in Education: A Systematic Review of Research and Applications. Educational Technology y Society, 17(4), 133-149.

Barba, R., Yasaca, S., y Manosalvas, C. (2015). Impacto de la realidad aumentada móvil en el proceso enseñanza-aprendizaje de estudiantes universitarios del área de medicina. Investigar con y para la Sociedad. Cádiz: Bubok Publishing S.L.

Barroso, J., y Cabero, J. (2010). La investigación educativa en TIC. Visiones prácticas. Madrid: Síntesis.

Bolliger, D. U., Supanakorn, S., y Boggs, C. (2010). Impact of podcasting on student motivation in the online learning environment. Computers \& Education, 55(2), 714-722. doi: 10.1016/j. compedu.2010.03.004.

Bongiovani, P. (2013). Realidad aumentada en la escuela: Tecnología, experiencias $e$ ideas. Recuperado de http://www. educacontic.es/blog/realidad

Bressler, D. M., y Bodzin, A. M. (2013). A mixed methods assessment of students' flow experiences during a mobile augmented reality science game. Journal of Computer Assisted Learning, 29(6), 505-517. doi: 10.1111/jcal.12008.

Cabero, J., y Barroso, J. (2016). The educational possibilities of Augmented Reality. NAER. New Approaches in Educational Research, 5(1), 44-50. doi: 10.7821/naer.2016.1.140.

Cabero, J., y García, F. (Coords.) (2016). Realidad aumentada. Tecnología para la formación. Madrid: Síntesis.

Cabero, J., y Llorente, M. C. (2009). Actitudes, satisfacción, rendimiento académico y comunicación online en procesos de formación universitaria en blended learning”. Revista Electrónica Teoría de la Educación: Educación y Cultura en la Sociedad de la Información. 1O(1). Recuperado de http://www.usal.es/ teoriaeducacion/ rev numero $10 \quad 01 /$ n10 01 cabero lorente.pdf

Cabero, J., y Marín, V. (2013). Valoración del entorno formativo universitario Dipro 2.o. Profesorado. Revista de Curriculum y formación del profesorado, 17(2), 369383.

Carozza, L., Tingdahi, D., Bosché, F., y Gool, L. (2014). Markerless VisionBased Augmented Reality for Urban Planning. Computer-Aided Civil and Infrastructure Engineering, 29(1), 2-17. doi: 10.1111/j.1467-8667.2012.00798.x.

Castaño, C., Maiz, I., y Garay, U. (2015). Diseño, motivación y rendimiento en un 
curso MOOC cooperativo. Comunicar, 44, 19-26.

Chang, H., Wu, K., y Hsu, Y. (2013). Integrating a mobile augmented reality activity to contextualize student learning of a socioscientific issue. British Journal of Educational Technology, 44(3), E95-E99. doi: 10.1111/j.1467-8535.2012.01379.x.

Che, Y. (2012). A study of learning effects on e-learning with interactive thematic video. Journal Educational Computing Research, 47(3), 279-292. doi: 10.2190/ EC.47.3.

Chen, C.-M., y Tsai, Y. (2012). Interactive augmented reality system for enhancing library instruction in elementary schools. Computers \& Education, 59, 638-652. doi: 10.1007/s10956-012-9405-9.

Cheng, Y., y Yeh, H. (2009). From concepts of motivation to its application in instructional design: Reconsidering motivation from an instructional design perspective. British Journal of Educational Technology, 40(4), 597-605. doi: 10.1111/j.1467-8535.2008.00857.x.

Cózar, R., De Moya, M., Hernández, J., y Hernández, J. (2015). Tecnologías emergentes para la enseñanza de las Ciencias Sociales. Una experiencia con el uso de Realidad Aumentada en la formación inicial de maestros. Digital Education Review, 27, 138-153.

Cubillo, J., Martín, S., Castro, M., y Colmenar, A. (2014). Recursos digitales autónomos mediante realidad aumentada. Revista Iberoamericana de Educación a Distancia, 17, 241-274.

De Pedro Carracedo, J., y Méndez, C. L. M. (2012). Realidad Aumentada: Una Alternativa Metodológica en la Educación Primaria Nicaragüense. IEEE-RITA, 7, 102-108. Recuperado de http://www.redusoi.org/docs/ LibroActasCAFVIR2011.pdf $\#$ page $=300$

Di Serio, A., Blanca, M., y Delgado, C. (2013). Impact of an augmented reality system on students' motivation for a visual art course.
Computers \& Education, 68, 586-596. doi: 10.1016/j.compedu.2012.03.002.

Düunser, A., Walker, L., Horner, H., y Bentall, D. (2012). Creating interactive physics education books with augmented reality. En V. Farrell, G. Farrell, C. Chua, W. Huang, R. Vasa \& C. Woodward (eds.), OzCHI'12, Proceedings of the 24th Australian Computer Human Interaction Conference (pp. 107-114). ACM: New York. doi: 10.1145/2414536.2414554.

Etxeberria, J., y Tejedor, J. (2005). Análisis descriptivo de datos en educación. Madrid: La Muralla.

Fombona, J., Pascual, M. J., y Madeira, M. F. (2012). Realidad aumentada, una evolución de las aplicaciones de los dispositivos móviles. Píxel-Bit. Revista de Medios y Educación, 41, 197-210.

Fonseca, D., Redondo, E., y Valls, F. (2016). Motivación y mejora académica utilizando realidad aumentada para el estudio de modelos tridimensionales arquitectónicos. Education in the Knowledge Society, EKS, 17(1), 45-64.

Gazcón, N. (2015). Libros Aumentados: Extensión del Concepto, Exploración e Interacciones. Bahía Blanca, Universidad Nacional del Sur, tesis doctoral no publicada.

Han, J., Jo, M., Hyun, E., y So, H. (2015). Examining young children's perception toward augmented reality-infused dramatic play. Education Technology Research Development, 63, 455-474.

Huang, W., Huang, W., y Tschopp, J. (2010). Sustaining iterative game playing processes in DGBL: The relationship between motivational processing and outcome processing. Computers \& Education, 55, 789-797. doi: 10.1016/j. compedu.2010.03.011.

Jamali, S., Fairuz, M. Wai, K., y Oskam, Ch. (2015). Utilising mobile-augmented reality for learning human anatomy. Procedia Social and Behavioral 
Sciences, 197, 659-668. doi: 10.1016/j. sbspro.2015.07.054.

Jeřábek, T., Rambousek, V., y Wildová, R. (2014). Specifics of Visual Perception of The Augmented Reality in The Context of Education. Procedia Social and Behavioral Sciences, 159, 598-604. doi: 10.1016/j.sbspro.2014.12.432.

Johnson, L., Adams, S., Cummins, M., Estrada, V., Freeman, A., y Hall, C. (2016). NMC Horizon Report: 2016 Higher Education Edition. Austin, Texas: The New Media Consortium. Recuperado de http://blog.educalab.es/intef/wpcontent/uploads/sites/4/2016/03/ Resumen Horizon Universidad 2016 INTEF mayo 2016.pdf

Kamarainen, A., Metcalf, Sh., Grotzer, T., Browne, A., Mazzuca, D., Tutwiler, M., y Dede,Ch.(2013).EcoMOBILE:Integrating augmented reality and probeware with environmental education field trips. Computers y Education, 68, 545-556. doi: 10.1016/j.compedu.2013.02.018.

Keller, J. M. (1983). Motivational design of instruction. In C. M. Reigeluth (Ed.), Instructional-design theories and models: An overview of their current status (386-434). Hillsdale, NJ: Lawrence Erlbaum Associates.

Keller, J. M. (1987). Strategies for stimulating the motivation to learn. Performance and Instruction, 26(8), 1-7.

Keller, J. M. (2010). Motivational design for learning and performance. New York: Springer Science+Business.

Kim. K., Hwang, J., y Zo, H. (2016): Understanding users' continuance intention toward smartphone augmented reality applications. Information Development, 32(2), 161-174.

Lin, T., Been-Lirn, H., Li, N., Wang, H., y Tsa, Ch. (2013). An investigation of learners' collaborative knowledge construction performances and behavior patterns in an augmented reality simulation system. Computers \& Education, 68, 314-
321. doi: http://dx.doi.org/10.1016/j. compedu.2013.05.011

Loorbach, N., Peters, O., Karreman, J., y Steehouder, M. (2015). Validation of the Instructional Materials Motivation Survey (IMMS) in a self-directed instructional setting aimed at working with technology. British Journal of Educational Technology, 46(1), 204-218. doi: 10.1111/ bjet.12138.

Lu, J., y Ying-Chieh, L. (2014). Integrating augmented reality technology to enhance children's learning in marine education. Environmental Education Research, 21(4), 525-541. doi: 10.1080/13504622.2014.911247.

$\mathrm{Lu}$, S., y Liu, Y-Ch. (2015). Integrating augmented reality technology to enhance children's learning in marine education. Environmental Education Research, 21(4), 525-541, doi: http://dx.doi.org/10. 1080/13504622.2014.911247

Marín, V., Cabero, J., y Barroso, J. (2014). Evaluando los entornos formativos online. El caso de DIPRO 2.o. REDU. Revista de docencia universitaria, 12(2), 375-399.

Mateo, J. (2004). La investigación expost-facto. En R. Bisquerra (coord.), Metodología de la investigación educativa (195-230). Madrid: La Muralla.

Mehmet, H. (2016). The classification of augmented reality books: a literature review. Proceedings of INTED2016 Conference, (pp. 4110-4118).Valencia: INTED.

Morales, M., Benítez, C., Silva, D., Altamirano, M., y Mendoza, H. M. (2016). Aplicación móvil para el aprendizaje del inglés utilizando realidad aumentada. Revista Iberoamericana de Producción Académica y Gestión Educativa, Recuperado de http://www.pag.org.mx/ index.php/PAG/article/viewFile/513/552

O'Dwyer, L., y Bernauer, J. (2014). Quantitative research for the qualitative researcher. California: Sage. 
Prendes, C. (2015). Realidad aumentada y educación: análisis de experiencias prácticas. Pixel-Bit. Revista de Medios y Educación, 46, 187-203. doi: 10.12795/ pixelbit.2015.i46.12.

Proske, A., Roscoe, R., y McNamara, D. (2014). Game-based practice versus traditional practice in computer-based writing strategy training: effects on motivation and achievement. Education Technology Research Development, 62, 481-505. doi: 10.1007/s11423-014-93492.

Sabariego, M. (2012). El proceso de investigación (parte 2). En R. Bisquerra (coord.), Metodología de la investigación educativa, ( $3^{\mathrm{a}}$ ed.), (127-163). Madrid: La Muralla.

Sans, A. (2012). Métodos de investigación de enfoque experimental. En R. Bisquerra (coord.), Metodología de la investigación educativa (151-193). ( ${ }^{\mathrm{a}}$ ed.). Madrid: La Muralla.

Santos, M. Wolde, A., Taketomi, T., Yamamoto, G., Rodrigo, M., Sandor, Ch., y Kato, H. (2016). Augmented reality as multimedia: the case for situated vocabulary learning. Research and Practice in Techology Enhanced Learning, 11(4), 1-23.

Sevillano, M. L., y Vázquez, E. (2015). Modelos de investigación en contextos ubicuos y móviles en educación superior. Madrid: McGraw-Hill/Uned.

Tecnológico de Monterrey (2015). Reporte EduTrends. Radar de Innovación Educativa 2015. Monterrey: Tecnológico de Monterrey.

Wei, X., Weng, D., Liu, Y., y Wang, Y. (2015). Teaching based on augmented reality for a technical creative design course. Computers \& Education, 81, 221-234. doi: 10.1016/j.compedu.2014.10.017.

Wojciechowski, R., y Cellary, W. (2013). Evaluation of learners' attitude toward learning in ARIES augmented reality environments. Computers \& Education, 68, 570-585. doi: 10.1016/j. compedu.2013.02.014.

Wu, H-S., Lee, S., Chang, H-Y., y Liang, J. (2013). Current status, opportunities and challenges of augmented reality in education. Computers \& Education, 62, 4149. doi: 10.1016/j.compedu.2012.10.024.

\section{PERFIL ACADÉMICO Y PROFESIONAL DE LOS AUTORES}

Julio Cabero Almenara. Catedrático de Tecnología Educativa de la Universidad de Sevilla, es director del Secretariado de Recursos Audiovisuales y Nuevas Tecnologías de la Universidad de Sevilla. Edito de la revista internacional Pixel Bit, Revista de Medios y Educación. Sus líneas de investigación giran en torno a las tecnologías emergentes.

E-mail: cabero@us.es

Bárbara Fernández Robles. Licenciada en Pedagogía por la Universidad de Sevilla. Posee Máster en formación y orientación profesional para el empleo. Actualmente estudiante de doctorado de la Universidad de Córdoba. Ha desempeñado funciones de asesoramiento, seguimiento y producción de contenidos digitales para la docencia en el Instituto Tecnológico de Santo Domingo. En este momento desarrolla esta labor en la Fundación Extensus.

E-mail: bfernandezrobles@gmail.com 


\section{DIRECCIÓN DE LOS AUTORES}

Facultad de Ciencias de la Educación

Calle Pirotecnia $\mathrm{s} / \mathrm{n}$

41018-Sevilla (España)

Verónica Marín Díaz. Profesora Titular de Universidad de la Facultad de Ciencias de la Educación de la Universidad de Córdoba, ha sido Directora del Máster en Educación Inclusiva de la citada universidad en el período 2012-2015. Miembro del grupo de investigación e2i de la Universidad de Córdoba y del Grupo de Tecnología Educativa de la Universidad de Sevilla. Editora de la revista internacional EDMETIC, Revista de Educación Mediática y TIC. Sus líneas de investigación giran en torno a las tecnologías emergentes.

E-mail: vmarin@uco.es

\section{DIRECCIÓN DE LA AUTORA}

Facultad de Ciencias de la Educación

Ada. San Alberto Magno s/n

14004-Córdoba (España)

Fecha de recepción del artículo: 07/10/2016

Fecha de aceptación del artículo: 02/01/2017

\section{Como citar este artículo:}

Cabero Almenara, J., Fernández Robles, B., y Marín Díaz, V. (2017). Dispositivos móviles y realidad aumentada en el aprendizaje del alumnado universitario. RIED. Revista Iberoamericana de Educación a Distancia, 20(2), pp. 167-185. doi: http:// dx.doi.org/10.5944/ried.20.2.17245 